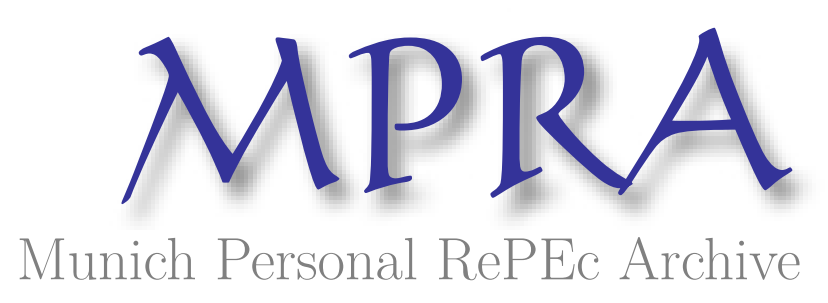

\title{
A Behavioral Model of Bubbles and Crashes
}

Kaizoji, Taisei (kaizoji@icu.ac.jp)

International Christian University

10 January 2010

Online at https://mpra.ub.uni-muenchen.de/20352/

MPRA Paper No. 20352, posted 02 Feb 2010 03:16 UTC 


\title{
A Behavioral Model of Bubbles and Crashes
}

\author{
Taisei Kaizoji ${ }^{1}$ \\ Department of Economics and Business \\ International Christian University \\ Tokyo, 181-8585, Japan
}

\begin{abstract}
The aim of this paper is to provide one potential theoretical explanation for questions how asset bubbles come about, why it persists, and what caused it to burst. We propose a new model of bubbles and crashes. We divide the risky assets into two classes, the bubble asset and the non-bubble asset, and the risk-free asset. Investors are divided into two groups, the rational investors and the noise traders. The rational investors maximize their expected utility of their wealth in the next period. Noise traders maximize their random utility of binary choice: holding the bubble asset and holding the risk-free asst.

We demonstrate that noise-traders' herd behavior, which follows the behavior getting a majority, occurs when the number of noise-traders increases, and their herd behavior gives cause to a bubble, and their momentum trading prolongs bubble. However, rising stock price slows down as the noise-trader's behavior approaches to a stationary state, so that the price momentum begins to decrease in the second half of bubble. We demonstrate that decreasing the price momentum lead to market crash.
\end{abstract}

\section{Introduction}

In the last few decades, the asset markets have been frequently visited by bubbles and the subsequent crashes. The increasingly frequent market crashes have

${ }^{1}$ E-,mail address : kaizoji@icu.ac.jp 
attracted the attention of academics and policy makers. Although many academics, practitioners and policy makers have studied questions related to collapsing asset price bubbles, the questions, how asset bubbles come about, why it persists, and what caused it to burst, have been the greatest myths. What is the origin of bubbles? Why asset prices are deviated away from fundamental value and what might have triggered the speculative mania?

Brunnermeier and Nagel (2004) extracted hedge fund holdings from Form 13F, including those of well-known managers such as Soros, Tiger, Tudor, and others in the period of the internet bubble ${ }^{2}$ from 1998 to 2000 . They found that, over the sample period 1998 to 2000 , many hedge fund managers tried to ride rather than attack bubbles, suggesting the existence of mechanisms that non-rational investors to surf bubbles rather than attempt to arbitrage them. Using manager age as a proxy for experience, Greenwood and Nagel (2008) study the portfolio decisions of experienced and inexperienced mutual fund managers during the internet bubble of 1998 to 2000. They found that at the start of the bubble, younger managers, who have not yet directly experienced the consequences of a stock market downturn, show little deviation from older managers, but leading up to the peak in March 2000, younger managers strongly increase their holdings of technology, while older managers do not. And then young managers decrease them during the downturn. Brennan (2004) also proposes that increased stock market participation by individuals with little investment experience may have been the driving factor of the internet bubbles. Another recent growing body of literature is also devoted to the existence of the momentum trading (also referred to as positive-feedback trading). Many empirical studies documents that the momentum in stock prices is positive in the short term, but eventually reversed in the long term. Among many literatures, the existence of the short-run momentum in stock prices is documented by Jegadeesh and Titman (1993), and the long-run reversal in stock prices is documented by DeBondt and Thaler (1985). Many researchers believe that the

\footnotetext{
${ }^{2}$ It is widely believed that the internet stocks were in the midst of stock price bubble in the 1999 to 2000 period. The internet bubble is investigated by Ofek and Richardson (2003), and Battalio and Schultz (2006).
} 
empirical evidences on the momentum trading (positive feedback trading) prove the existence of the traders' herd behavior, and herding have potential to explain speculative bubbles (see for example, DeLong et al. (1990)). These empirical findings are consistent with experiments in laboratory asset markets conducted. Smith, Suchanek, and Williams (1988) find that (a) bubbles and crashes occur regularly in laboratory asset markets when market participants are inexperienced, but (b) price gradually approach fundamentals when the participants, who have experienced bubbles and crashes in prior trading sessions, interact repeatedly in similar markets. Haruvy, Lahav, and Noussair (2009) finds that the investors' expectations of prices are adaptive, and primarily based on past trends in the current and previous markets in which they have participated. Most traders do not anticipate market downturns the first time they participate in a market, and are more prone to the optimism that fuels the bubble. In the opposite direction, when experienced, they typically overestimate the time remaining before market peaks and downturns occur. In summary, the studies mentioned above indicate that the bubble is caused by the non-rational investors who attempt to surf bubble.

In this paper we propose a model to explore a mechanism of the subsequent collapse of bubble. Our model has several advantages that are absent from popolar models of asset bubbles (for examples, DeLong et al. (1990), and Abreu and Brunnermeier (2003)). We consider that investors trade three assets: the bubble asset the non-bubble asset, and the risk-free asset. Investors are divided into two groups, rational investors and noise traders. The rational investors are corresponded to experienced managers who have directly experienced the consequences of asset market crashes while noise traders are young managers who have not yet directly experienced the consequences of a stock market downturn. In accordance with traditional asst-pricing models (see e.g., Mossin (1966) and Lintner (1969)), the rational investors chooses that the portfolio of three assets, bubble asset, non-bubble asset and the risk-free asset which will maximize his expected utility of end-of-period wealth. On the other hand, noise traders maximize their random 
utility $^{3}$ of alternatives, that is, holding bubble stocks and holding the risk-free asset. We assume that a noise trader's decision-making is influenced by (i) the decisions of the other noise-traders, and (ii) the return momentum on the bubble asset which is defined as the exponential moving average of the price changes. The noise-trader's utility function of an alternative is composed of those two attributes, and random variable. In our model, we show that as the interaction among noise traders is strengthened, the extent, that each noise-trader is influenced by the decisions of other noise-traders, is reinforced, and noise traders begin to follow the herd. In the attribute (ii), we also assume that the noise-traders' expectation of bubble stock prices is adaptive. The noise-traders adapt the positive feed-back strategy (momentum strategy) on the bubble asset. Our model indicates a mechanism that that noise-traders' herd behavior, which follows the behavior getting a majority, and their momentum trading, gives cause to a bubble ended up with a crash.

The paper proceeds as follows. The model is described in Section 2. In Section 3, and in Section 4 we give a theoretical explanation on a mechanism of bubble and crash. We give concluding remarks in Section 5.

\section{Model}

Consider the risky assets, and the risk-free asset labeled $f$. We divided the risky assets into two classes: the bubble asset labeled 1 and the non-bubble asset labeled 2 which are portfolios of stocks in corresponding sectors. We also divide into two groups of investors with different decision making. The first group of investors is a group of rational investors who maximize their expected utility of wealth in the next period. The second group of investors is the group of the noise-traders who maximize the random utility of the binary choice: holding the bubble asset and holding the risk-free asset, and trades the bubble asst and the risk-free asset.

\footnotetext{
${ }^{3}$ The qualitative choice models based on maximization of the agent's random utility has been developed by McFadden (1974) .
} 


\subsection{Rational investors}

Let us consider the behavior of rational-investors. We shall assume that there is a number $M$ of rational investors. Their object is to maximize the expected utility $E U\left(W_{t+1}\right)$ of wealth $W_{t+1}$ in the next period, $\mathbf{t}+1$ by selecting a portfolio mix of the two different types of the risky assets and the risk-free asset labeled $f$. We assume that rational investor's preferences are characterized by the constant-absolute risk aversion (CARA) utility with the coefficient of risk aversion, $\gamma$. The rational investors are assumed to be identical. We consider the behavior of the representative-rational investor hereafter. The maximization problem which the rational investors solve is equivalent to the mean-variance model. That is, in his choice among all the possible portfolios, the rational investor is satisfied to be guided by its expected yields $E\left(W_{t}\right)$ and its variance $V\left(W_{t}\right)$.

$$
\begin{aligned}
& \underset{x_{1 t}, x_{2 t}, x_{f t}}{\operatorname{Max}} \operatorname{EU}\left(W_{t+1}\right)=\operatorname{Max}\left\{E\left(W_{t}\right)-\frac{\gamma}{2} V\left(W_{t}\right)\right\} \\
& \text { s.t. } \quad p_{1 t}\left(x_{1 t}-x_{1 t-1}\right)+p_{2 t}\left(x_{2 t}-x_{2 t-1}\right)+q\left(x_{f t}-x_{f t-1}\right)=0
\end{aligned}
$$

where an investor's wealth is written as $W_{t}=\left(p_{1 t} x_{1 t}+p_{2 t} x_{2 t}+p_{f t} x_{f t}\right)$ where $x_{j t}$ denotes the demand for the asset $j$ in the period $t$, and $p_{j t}$ denotes the unit price of the asset $j$ during the period $t$, and $q$ denotes the unit price of a risk-free asset. We assume without loss of generality that $q$ is constant for all $t$. The expected value of the wealth $E\left(W_{t+1}\right)$, and the variance of the wealth $V\left(W_{t+1}\right)$ is defined as

$$
\begin{aligned}
& E\left(W_{t+1}\right)=x_{1 t} E\left(p_{1 t+1}\right)+x_{2 t} E\left(p_{2 t+1}\right)+x_{f t}, \\
& V\left(W_{t+1}\right)=\sigma_{1}^{2}\left(x_{1 t}\right)^{2}+\rho^{12} x_{1 t} x_{2 t}+\rho^{21} x_{2 t} x_{1 t}+\sigma_{2}^{2}\left(x_{2 t}\right)^{2}
\end{aligned}
$$

where $E\left(p_{j t+1}\right)$ is the expected value of $p_{j t+1}(j=1,2), \sigma_{j}^{2}$ the variance of $p_{j t+1}$, and $\rho^{i j}$ the covariance of $p_{i t+1}$ and $p_{j t+1}$.

The corresponding first-order conditions are: 


$$
\begin{aligned}
& \begin{aligned}
\frac{\partial E U}{\partial x_{1 t}} & =E\left(p_{1 t+1}\right)-\frac{\gamma}{2}\left[2 \sigma_{1}^{2} x_{1 t}+\rho^{12} x_{2 t}+\rho^{21} x_{2 t}\right]+\mu p_{1 t} \\
& =E\left(p_{1 t+1}\right)-\gamma\left[\sigma_{1}^{2} x_{1 t}+\rho^{12} x_{2 t}\right]+\mu p_{1 t}=0
\end{aligned} \\
& \frac{\partial E U}{\partial x_{2 t}}=E\left(p_{2 t+1}\right)-\gamma\left[\rho^{21} x_{1 t}+\sigma_{2}^{2} x_{2 t}\right]+\mu p_{2 t}=0 \\
& \frac{\partial E U}{\partial x_{f}}=1+\mu q=0 \\
& \frac{\partial E U}{\partial \mu}=p_{1 t}\left(x_{1 t}-x_{1 t-1}\right)+p_{2 t}\left(x_{2 t}-x_{2 t-1}\right)+\left(x_{f t}-x_{f t-1}\right)=0
\end{aligned}
$$

where $\mu$ denotes the Lagrangian. Demands for the bubble asset and non-bubble asset of the optimal portfolio are:

$$
\begin{aligned}
& x_{1 t}=\frac{1}{|A| \gamma}\left\{\left(E\left(p_{1 t+1}\right)-\frac{p_{1 t}}{q}\right) \sigma_{2}^{2}-\left(E\left(p_{2 t+1}\right)-\frac{p_{2 t}}{q}\right) \rho^{12}\right\} \\
& x_{2 t}=\frac{1}{|A| \gamma}\left\{\left(E\left(p_{2 t+1}\right)-\frac{p_{2 t}}{q}\right) \sigma_{1}^{2}-\left(E\left(p_{1 t+1}\right)-\frac{p_{1 t}}{q}\right) \rho^{21}\right\} \\
& x_{f t}=x_{f t-1}+\frac{p_{1 t}}{q}\left(x_{1 t}-x_{1 t-1}\right)+\frac{p_{2 t}}{q}\left(x_{2 t}-x_{2 t-1}\right) \\
& \text { where }|A|=\left|\begin{array}{cc}
\sigma_{1}^{2} & \rho^{12} \\
\rho^{21} & \sigma_{2}^{2}
\end{array}\right| .
\end{aligned}
$$

From equations (3), the expected risk premium is calculated as

$$
E\left[r_{1 t+1}\right]-r_{f}=\left(\frac{E\left(p_{1 t+1}\right)-p_{1 t} / q}{p_{1 t}}\right), E\left[r_{2 t+1}\right]-r_{f}=\left(\frac{E\left(p_{2 t}\right)-p_{2 t} / q}{p_{2 t}}\right) \text {. }
$$

Therefore, the rational investors' transaction depends on the expected risk premiums of risky assets. The excess demands for three assets by rational investors are calculated by subtracting $x_{j t}$ from $x_{j t+1}$ 


$$
\left\{\begin{array}{l}
x_{1 t}-x_{1 t-1}=\frac{1}{\gamma|A|}\left\{\left(E\left(p_{1 t+1}\right)-E\left(p_{1 t}\right)-\frac{\left(p_{1 t}-p_{1 t-1}\right)}{q}\right) \sigma_{2}^{2}-\left(E\left(p_{2 t+1}\right)-E\left(p_{2 t}\right)-\frac{\left(p_{2 t}-p_{2 t-1}\right)}{q}\right) \rho^{12}\right\} \\
x_{2 t}-x_{2 t-1}=\frac{1}{\gamma|A|}\left\{\left(E\left(p_{2 t+1}\right)-E\left(p_{2 t}\right)-\frac{\left(p_{2 t}-p_{2 t-1}\right)}{q}\right) \sigma_{1}^{2}-\left(E\left(p_{1 t+1}\right)-E\left(p_{1 t}\right)-\frac{\left(p_{1 t}-p_{1 t-1}\right)}{q}\right) \rho^{21}\right\} \\
x_{f t}-x_{f t-1}=\frac{p_{1 t}}{q}\left(x_{1 t}-x_{1 t-1}\right)+\frac{p_{2 t}}{q}\left(x_{2 t}-x_{2 t-1}\right)
\end{array}\right.
$$

The aggregated excess demands for the risky assets by rational investors are obtained by multiplying the number $M$ of rational investors:

$$
\left\{\begin{array}{l}
M \Delta x_{1 t}=\frac{M}{\gamma|A|}\left[\sigma_{2}^{2}\left(E\left(\Delta p_{1 t+1}\right)-\Delta p_{1 t} / q\right)-\rho^{12}\left(E\left(\Delta p_{2 t+1}\right)-\Delta p_{2 t} / q\right)\right] \\
M \Delta x_{2 t}=\frac{M}{\gamma|A|}\left[\sigma_{1}^{2}\left(E\left(\Delta p_{2 t+1}\right)-\Delta p_{2 t} / q\right)-\rho^{21}\left(E\left(\Delta p_{1 t+1}\right)-\Delta p_{1 t} / q\right)\right]
\end{array}\right.
$$

where $\Delta x_{i t}=x_{i t}-x_{i t-1}, \Delta p_{j t+1}=p_{j t+1}-p_{j t}$ and $E\left(\Delta p_{j t+1}\right)=E\left(p_{j t+1}\right)-E\left(p_{j t}\right)$

\subsection{Noise traders}

There is a large number $N$ of noise traders who is inexperienced and have not yet directly experienced the consequences of a market downturn. Each noise-trader is assumed to choose the bubble asset or the risk-free asset for each period. That is, each of them is a holder of the bubble asset or a holder of the risk-free asset.

We consider that the individual noise trader maximizes his/her random utility of the alternatives, that is, holding the bubble asset and holding the risk-free asset. The noise trader chooses an asset with the highest utility ${ }^{4}$. The noise-trader's random utility function is composed of the deterministic part which is assumed to represent average behavior, and a nondeterministic part to represent random deviations from this average. The random utility of alternatives is given as:

\footnotetext{
${ }^{4}$ The random utility function of discrete choice developed by McFadden (1974) who has developed qualitative choice models based on maximization of the agent's random utility.
} 


$$
\left\{\begin{array}{l}
U_{+}=\bar{U}_{+}+\varepsilon_{+} \\
U_{-}=\bar{U}_{-}+\varepsilon_{-}
\end{array} .\right.
$$

where $\varepsilon_{i}$ is a random variable. The noise trader attaches a value, $U_{i}$ to each of two alternatives, that is, holding the bubble stocks (labeled +) and holding the risk-free asset (labeled -).

A common procedure used in both economics and finance is to assume the existence of a "representative" or "average" individual who is assumed to have tastes equal to the average over all decision makers. Two possible explanations for the stochastic term are given by Hausman and Wise (1978). The first is that a noise trader behaves randomly, perhaps due to random firing of neurons; so that faced repeatedly with the same alternative set. The same individual makes different choices. Second is that there are unobserved characteristics of the individual and unobserved attributes of the alternatives. Given the specification of the utility function, each noise trader is assumed to choose the alternative that maximizes his utility. The maximization of the random utility gives the probability with which each alternative is chosen. The probability that he chooses each alternative is given as :

$$
\left\{\begin{array}{l}
P_{+}=\operatorname{Pr}\left[U_{+}>U_{-}\right]=\operatorname{Pr}\left[\bar{U}_{+}-\bar{U}_{-} \geq \varepsilon_{-}-\varepsilon_{+}\right] \\
P_{-}=\operatorname{Pr}\left[U_{-}>U_{+}\right]=\operatorname{Pr}\left[\bar{U}_{-}-\bar{U}_{+} \geq \varepsilon_{+}-\varepsilon_{-}\right]
\end{array}\right.
$$

where $P_{+}+P_{-}=1$.

We assume that individual noise trader's decision-making is influenced by (i) the other noise-traders and (ii) the return momentum on the bubble asset. To describe a noise-trader's utility function which is composed of those determinants, let us introduce a new variable $s$ that denotes the normalized excess of the bubble-asset holders over holders of risk-free asset which is defined as $s_{t}=\left(n_{+t}-n_{-t}\right) / N$ where $n_{+t}$ is the number of bubble stocks holders, $n_{-t}$ is number of traders, possessing by risk-free asset in the period $t$. Obviously $n_{+t}+n_{-t} \equiv N$. Using the variable $s_{t}$, we rewrite the equation $(8)$, 


$$
\left\{\begin{array}{l}
\bar{U}_{+}=\lambda s_{t}+H_{t} \\
\bar{U}_{-}=-\left(\lambda s_{t}+H_{t}\right)
\end{array}\right.
$$

where the parameter $\lambda$ is assumed to be positive and constant. Given $\lambda$ is a positive, an increase in $s_{t}$ raises the utility of holding the bubble asset, and reduces the utility of holding the risk-free asset in the direction of the minority decision. This means that the noise trader has a tendency to be in favor of the majority decision. $H_{t}$ denotes the return momentum of the bubble asset which is defined as the exponential moving average of the risk premium of the bubble asset,

$$
H_{t}=\theta \sum_{i=0}^{\infty}(1-\theta)^{i}\left(r_{t-i}-r_{f}\right) \text {. }
$$

where $r_{f}$ denotes the interest rate of the risk free asset. The return momentum $H_{t}$ of the bubble asset is equivalent to

$$
H_{t}=(1-\theta) H_{t-1}+\theta\left(r_{t-1}-r_{f}\right), \quad H_{t=0}=H_{0}
$$

where $0<\theta<1$. It means that the noise-trader's expectation on the risk premium is adaptive.

As the return momentum $H_{t}$ of the bubble asset is higher, the utility of holding the bubble asset raises, and the utility of holding the risk-free asset is reduced. The equations (10), (11) means that the noise-traders adapt momentum strategies which are a strategy that buys assets with high capital gains and sells assets with poor capital gains over the previous periods ${ }^{5}$.

McFadden (1974) has shown that if the random variable $\varepsilon_{i}$ are independently and identically distributed with the Gumbell distribution

$$
F(\varepsilon) \equiv \operatorname{Pr}\left[\varepsilon_{i} \leq \varepsilon\right]=\exp [-\exp [-\varepsilon]] .
$$

The probability that a utility-maximizing noise trader will choose each alternative, is expressed as:

\footnotetext{
${ }^{5}$ The fact that momentum strategies yield significant profits, have been well investigated. Jegadeesh and Titman (1993) examine a variety of momentum strategies and document that strategies earn profits.
} 


$$
\left\{\begin{array}{l}
P_{+}=\frac{\exp \left[\bar{U}_{+}\right]}{\exp \left[\bar{U}_{+}\right]+\exp \left[\bar{U}_{-}\right]} \\
P_{-}=\frac{\exp \left[\bar{U}_{-}\right]}{\exp \left[\bar{U}_{+}\right]+\exp \left[\bar{U}_{-}\right]}
\end{array}\right.
$$

Now we introduce a variable $v$ which is the probability that a transition is attempted by one of the noise-traders, and follows a uniform distribution over noisetraders. We assume that one noise-trader attempts a trade in one time unit. The individual transition probabilities per an unit time period is described as

$$
\left\{\begin{array}{l}
p_{\downarrow}\left(s_{t}\right)=v \cdot \frac{\exp \left[-\left(\lambda s_{t}+H_{t}\right)\right]}{\exp \left[\lambda s_{t}+H_{t}\right]+\exp \left[-\left(\lambda s_{t}+H_{t}\right)\right]} \\
p_{\uparrow}\left(s_{t}\right)=v \cdot \frac{\exp \left[\lambda s_{t}+H_{t}\right]}{\exp \left[\lambda s_{t}+H_{t}\right]+\exp \left[-\left(\lambda s_{t}+H_{t}\right)\right]}
\end{array}\right.
$$

The random variable $v$ determines the time scale in which a transition which is attempted by a noise trader occurs.

$p_{\downarrow}\left(s_{t}\right)$ is the transition probability that one of the noise traders who hold the bubble asset sells his bubble asset, and alternatively hold the risk-free asst. Inversely $p_{\uparrow}\left(s_{t}\right)$ the transition probability that one of the noise traders who hold the risk-free asset sells his risk-free asset and hold the bubble asset.

The effects of $\lambda$ and $H_{t}$ on the transition probabilities can be described as follows:

i) A positive $\lambda$ enlarges the transition probability in favor of the majority choice and reduces the transition probability in the direction of the minority choice. This positive feedback effect grows for a growing imbalance of choices.

ii) A positive momentum $H_{t}$ increases the probability that a noise trader changes from holding the risk-free asset to holding the bubble asset, and reduces the probability of changing from holding the bubble asset to holding the risk-free asset, and vice versa for negative $H_{t}$. 
Using the transition probabilities, the equation for the distribution $p\left(s_{t}\right)$ of stochastic process of $s$ is described by

$$
\begin{aligned}
p\left(s_{t+1}\right)-p\left(s_{t}\right)=[ & \left.w_{\downarrow}\left(s_{t}+\Delta s\right) p\left(s_{t}+\Delta s\right)-w_{\downarrow}\left(s_{t}\right) p\left(s_{t}\right)\right] \\
& +\left[w_{\uparrow}\left(s_{t}-\Delta s\right) p\left(s_{t}-\Delta s\right)-w_{\uparrow}\left(s_{t}\right) p\left(s_{t}\right)\right]
\end{aligned}
$$

where

$$
\left\{\begin{array}{l}
w\left[\left(s_{t}-\Delta s\right) \leftarrow s_{t}\right] \equiv w_{\downarrow}\left(s_{t}\right)=n_{t}^{-} p_{\downarrow}\left(s_{t}\right)=\frac{N}{2}\left(1+s_{t}\right) p_{\downarrow}\left(s_{t}\right) \\
w_{\uparrow}\left(s_{t}\right) \equiv w\left(\left(s_{t}+\Delta s\right) \leftarrow s_{t}\right)=n_{t}^{+} p_{\uparrow}\left(s_{t}\right)=\frac{N}{2}\left(1-s_{t}\right) p_{\uparrow}\left(s_{t}\right) \\
w_{\downarrow}\left(s_{t}+\Delta s\right) \equiv w\left(s_{t} \leftarrow\left(s_{t}+\Delta s\right)\right)=n_{t}^{-} p_{\downarrow}\left(s_{t}+\Delta s\right)=\frac{N}{2}\left(1+\left(s_{t}+\Delta s\right)\right) p_{\downarrow}\left(s_{t}+\Delta s\right) \\
w_{\uparrow}\left(s_{t}-\Delta s\right) \equiv w\left(s_{t} \leftarrow\left(s_{t}-\Delta s\right)\right)=n_{t}^{+} p_{\uparrow}\left(s_{t}-\Delta s\right)=\frac{N}{2}\left(1-\left(s_{t}-\Delta s\right)\right) p_{\uparrow}\left(s_{t}-\Delta s\right) \\
w\left(s_{t}^{\prime} \leftarrow s_{t}\right)=0 \quad \text { for } \quad s_{t}^{\prime} \neq s_{t} \pm \Delta s
\end{array}\right.
$$

where $\Delta s=N / 2$.

The equation (15) is called as the master equation (See Gardiner (1985)). When the number of noise traders is large, the equation (15) is equivalent to the dynamic equation of the mean $\hat{s}_{t}$ of $p\left(s_{t}\right)$ (see Weidlich and Haag (1983)),

$$
\Delta \hat{s}_{t+1} \equiv \hat{s}_{t+1}-\hat{s}_{t}=v\left[\tanh \left(\lambda \hat{s}_{t}+H_{t}\right)-\hat{s}_{t}\right]
$$

where $\hat{s}_{t}=\left(\hat{n}_{t}^{+}-\hat{n}_{t}^{-}\right) / N \cdot \hat{n}_{t}^{+}$denotes the number of noise traders who hold the bubble asset at the period $t$, and $\hat{n}_{t}^{-}$denotes the number of noise traders who hold the risk free asset. The solution of (17) corresponds to the maximum of the stationary distribution $p_{s t}\left(s_{t}\right)$ of the master equation (15), and the equation of (17) describes the collective behavior of the representative noise-trader.

Using the difference of $\hat{s}_{t}$ from period $t$ to period $t+1$, the aggregate excess demand for the bubble asset by all noise traders is defined as 


$$
Q\left[\hat{n}_{t}^{+}-\hat{n}_{t-1}^{+}\right]=\frac{Q N}{2}\left(\hat{s}_{t}-\hat{s}_{t-1}\right)
$$

where the parameter $Q$ denotes the number of shares of the bubble asset which is exchanged in any transaction by a noise trader, and is assumed to be constant. The equation (18) will be utilized when the market prices of the risky assets are calculated under the market clearing conditions in section 2.4 .

\subsection{Collective behavior of the representative noise-traders}

Before we discuss about bubbles and crashes, we describe the collective behavior of the representative noise-traders using equation (17). As the above conditions i) and ii) indicate, the noise-trader's transition probability depends on the variables $\lambda$ and $H_{t}$. For simplicity of analysis, let us assume that the return momentum $H_{t}$ is a parameter $H$. The solutions of the mean equation (17) can be summarized with respect to $\lambda$ and $H$ as follows:

i) The case of $0<\lambda<1$ and arbitrary $H$ :

There is only one possible solution $s^{* *}$. The solution corresponds to the maximum of the stationary distribution $p_{s t}\left(s_{t}\right)$. For $H_{t}=0$ and $0<\lambda<1$, the only one possible solution is zero.

In this case which the relatively small number of the noise-traders participate into trade, the driving force of the collective behavior of noisetraders mainly the return momentum $H$ but strong herding among noisetraders dose not function. In Figure 1 the graphical solution to (17) is plotted for $\lambda<1$ and the different values of $H$. When the return momentum $H$ is positive (negative), the solution moves from zero to a positive value (a negative value).

ii) The case of $\lambda>1$ and $|H|<\bar{H}$ : 
$\bar{H}$ is determined by the equation $\cosh ^{2}[\bar{H}-\sqrt{\lambda(\lambda-1)}]=\lambda$. There are three possible solutions $s^{*}<s^{* *}<s^{* * *}$. The solution $s^{*}$ and $s^{* * *}$ are called the bearmarket equilibrium and the bull-market equilibrium respectively. Therefore, as the parameter $\lambda$ increases, the solution $s^{* *}$ is unstable, and appears the bear-market equilibrium $s^{*}(<0)$ and bull-market equilibrium $s^{* * *}(>0)$. As $\lambda$ exceeds unity, the stationary distribution $p_{s t}\left(s_{t}\right)$ is from unimodal to multimodal. This bifurcation is called as the secondorder phase transition. In Figure 2 the graphical solution to (17) is plotted for $\lambda>1$.

iii) The case of $\lambda>1$ and $|H|=\bar{H}$ :

Two of the tree solutions $s^{*}<s^{* *}<s^{* * *}$ coincide at $s_{c}= \pm \sqrt{(\lambda-1) / \lambda}$.

An increase (a decrease) in the return momentum $H$ causes the curve which indicates the transcendental equation (17) to shift up (down), so that the solutions rise (fall). Figure 3 shows the states that two of the tree solutions coincide.

iv) There case of $\lambda>1$ and $|H|>\bar{H}$ :

There is one solution again. When $\lambda>1$, and $H$ is negative and decreasing continuously, the stationary distribution $p_{s t}\left(s_{t}\right)$ is from multimodal to unimodal. The solution jumps down from $s^{* * *}$ to $s^{*}$ at the moment that the return moment $H$ falls below $-\bar{H}$. Inversely, the solution jumps up from $s^{*}$ to $s^{* * *}$ at the moment that or that the return moment $H$ exceeds $+\bar{H}$. This bifurcation is called as the first-order phase transition.

\subsection{Market-clearing prices}


The market clearing condition requires that the aggregated excess demand (supply) for each asset by rational investors is equal to the aggregated excess demand (supply) by noise traders from the period t-1 to the period t. That is, if one noisetrader changes from a holder of the risk-free asset to a holder of the bubble stocks, then the prices are adjusted such that rational investors supply the corresponding number of the bubble stocks. The market clearing conditions are described as

$$
\left\{\begin{array}{l}
M \Delta x_{1 t}+\frac{Q N}{2} \Delta \hat{s}_{t t}=\frac{M}{\gamma|A|}\left[\sigma_{2}^{2}\left(E\left(\Delta p_{1 t+1}\right)-\frac{\Delta p_{1 t}}{q}\right)-\rho^{12}\left(E\left(\Delta p_{2 t+1}\right)-\frac{\Delta p_{2 t}}{q}\right)\right]+\frac{Q N}{2} \Delta \hat{s}_{t}=0 \\
M \Delta x_{2 t}=\frac{M}{\gamma|A|}\left[-\rho^{12}\left(E\left(\Delta p_{1 t+1}\right)-\frac{\Delta p_{1 t}}{q}\right)+\sigma_{1}^{2}\left(E\left(\Delta p_{2 t+1}\right)-\frac{\Delta p_{2 t}}{q}\right)\right]=0
\end{array}\right.
$$

Solving the equations (19) with respect to the price changes on the risky assets $\Delta p_{i t},(i=1,2)$ we can obtain the price changes of the risky assets which satisfy the market-clearing conditions. In summary, the dynamics of stock markets can be described as:

$$
\left\{\begin{array}{l}
\Delta p_{1 t} \equiv p_{1 t}-p_{1 t-1}=q\left[\gamma \sigma_{1}^{2} \kappa \Delta \hat{s}_{t}+E\left(\Delta p_{1 t+1}\right)\right] \\
\Delta p_{2 t} \equiv p_{2 t}-p_{2 t-1}=q\left[\gamma \rho^{12} \kappa \Delta \hat{s}_{t}+E\left(\Delta p_{2 t+1}\right)\right] \\
\Delta \hat{s}_{t} \equiv \hat{s}_{t}-\hat{s}_{t-1}=v\left[\tanh \left(\lambda \hat{s}_{t-1}+H_{t}\right)-\hat{s}_{t-1}\right] \\
\Delta H_{t} \equiv H_{t}-H_{t-1}=\theta\left[\left(r_{t-1}-r_{f}\right)-H_{t-1}\right]
\end{array}\right.
$$

where $\kappa=\frac{Q N}{2 M}$. We assume that the term $E\left(\Delta p_{j t+1}\right)$, which is the change of the fundamental prices of the risky assets. The terms are often considered as a random variable which fluctuates. However, for simplicity of analysis, we consider the terms are constant over time, that is, $E\left(\Delta p_{j t+1}\right)=0,(j=1,2)$. Then, the price changes of the risky assets depend completely on $\Delta \hat{s}_{t}$. Since $q \gamma \sigma_{1}^{2} \kappa>0$, the price change $\Delta p_{1 t}$ of the bubble asset increases (decreases) proportionally with respect to $\Delta \hat{s}_{t}$, and for $\rho^{12}>0\left(\right.$ for $\left.\rho^{12}<0\right)$, the price change $\Delta p_{1 t}$ of the non-bubble asset is positively (negatively) correlated with $\Delta \hat{s}_{t}$. If one noise-trader changes from a holder of the 
risk-free asset to a holder of the bubble asset, then the price $p_{1 t}$ of the bubble asset rises by $q \gamma \sigma_{1}^{2} \times \frac{Q}{M}>0$, and the price $p_{2 t}$ of the non-bubble asset rises (falls) by $q \gamma \rho^{12} \times \frac{Q}{M}$ for $\rho^{12}>0\left(\rho^{12}<0\right)$

\section{How does bubble come about?}

As discussed in subsection 2.3., when the parameter $\lambda$ exceeds unity, the unique solution $s^{* *}(=0)$ is unstable, and appears newly two solutions, the bear-market solution $s^{*}(<0)$ and bull-market solution $s^{* * *}(>0)$ both of which are stable, under $H_{t}=0$. (See Figure 4.) Let us consider the motion of the prices of the bubble asset starting from the unstable solution $s^{* *}(=0)$. Depending on the value of $\hat{s}_{0}$ at the initial time, the bubble asset can either enter a bull market or a bear market. That is, when the initial value of $\hat{s}_{0}$ is positive, the price of the bubble asset raise, and enter a bubble phase, and visa versa. Run-up in the bubble-asset price $p_{1 t}$ is due to increases in the noise-traders' excess demand $\left(Q N \Delta \hat{s}_{t} / 2\right)$ for the bubble asset, and it increases the return momentum $H_{t}$. The increases in the momentum $H_{t}$ next pull up toward the bull-market solution $s^{* * *}(>0)$. The aggregate demand for the bubble asset by noise-traders is increased further due to the noise traders' herding. As the noise-traders' excess demand for the bubble asset is positive and increases, the price $p_{1 t}$ of the bubble asset increases and is over-evaluated. Thus, the momentum $H_{t}$ increases in the first half of bubbles. This inflationary spiral gives cause to the asset bubble. For $\lambda>1$ and $H_{t}>\bar{H}$ the bear-market equilibrium disappears, and the bull-market equilibrium is unique and stable. Thus, the bubble persists until the imbalance of buyers and sellers over the noise traders, $\hat{s}_{t}$ approaches to the bull-market equilibrium $s^{* * *}(>0)$. (See Figure 5.) 
The expected risk premium is described as

$E\left[r_{1 t+1}\right]-r_{f}=\left(\frac{E\left(p_{1 t+1}\right)-p_{1 t} / q}{p_{1 t}}\right)$ and $E\left[r_{2 t+1}\right]-r_{f}=\left(\frac{E\left(p_{2 t}\right)-p_{2 t} / q}{p_{2 t}}\right)$

In the period of bubbles, the actual high return on the bubble asset is earned as the result of low expected future returns. That is, rational investors sell the bubble asset because of the lower expected risk premium, and in the opposite direction, more noise traders buy the bubble asset more due to rises in the utility of holding the bubble asset. As a result, the noise traders get a capital gain from the price run-up in the period of bubbles. The actual price change $\Delta p_{1 t}$ of the bubble asset is contrary to the rational investors' expectation in the period of bubbles.

Many recent academic articles have argued that after the increases in stock prices over the last decade, the expected equity premium is low and perhaps negative (Lee, Myers and Swaminathan (1999); Fama and French (2000); Shiller (2000)). Our model gives a persuasive explanation on the risk premium puzzle.

Then, consider the case that the bubble asset has a weak positive correlation with the non-bubble asset, $\rho^{12}>0$ historically. As the noise-traders' excess demand for the bubble asset increases, the price of the non-bubble asset $p_{2 t}$ increases slightly reflecting the weak positive-correlation.

\section{Why do bubbles burst?}

In the first half of bubbles, the noise-traders' excess demand for the bubble asset is sharply increasing, so that the price of the bubble asset is also sharply increasing, but in the second half of bubbles, as the noise-traders' imbalance $\hat{s}_{t}$ excess demand for the bubble asset is approaching the bull market equilibrium $s^{* * *}$, the noisetraders' excess demand $\Delta \hat{s}_{t}$ excess demand for the bubble asset is approaching zero, and so a rise in the bubble-asset price slows down. 
Decreasing the momentum $H_{t}$ changes the bull-market equilibrium $s^{* * *}$ downward, so that the noise traders' excess demand $\Delta \hat{s}_{t}$ for the bubble asset declines, and the bubble-asset price starts to decrease. As the momentum $H_{t}$ decreases, the bear-market equilibrium $s^{*}$ appears again. This deflationary spiral continues to decrease and become negative in its final stage of bubbles. until. A crash can be suddenly caused by a trifling bad in the end stage of bubbles when the return momentum $H_{t}$ declines until $H_{t}=-\bar{H}$. In an instant when $H_{t}$ falls below $-\bar{H}$, the probability of the noise trader's selling the bubble asset is higher than the probability of the noise trader's buying the bubble asset. In our model the market crash is considered as the so called first-order phase transition. (See Figure 5.) The noise traders' selling on balance in the period of a crash depends on the parameter $\lambda$. The noise-traders' panic selling of the bubble asset give also rise to the decline in the price of non-bubble asset reflecting a historically positive correlation, $\rho^{12}>0$. Therefore, the crash is a "contagious" market-wide phenomenon.

After a crash, the rational investors buy the bubble asset, which they sell and/or went short in the period of bubbles, back when the expected risk premium (21) is positive. After all, the rational investors can make a profit from a long-term investment, while the noise-traders lose money.

\section{Concluding Remarks}

This paper provides one potential theoretical explanation for asset bubble and crash. A merit of this paper is to propose that a model describing the rationality of the noise trader's behavior, and a mechanism of bubble and carash which is caused by the noise trader's behavior.

\section{References:}


Abreu, D., and M. K. Brunnermeier, (2003), Bubbles and Crashes, Econometrica 71, 173-204.

Brennan, Michael, (2004), How Did It Happen?, Economic Notes 33, 3-22.

Brunnermeier, Markus and Stefan Nagel, (2004), Hedge funds and the technology bubble, Journal of Finance, 59 (5), 2013 - 2040.

DeBondt, W. F.M., and Richard Thaler, (1985), Does the stock market overreact? Journal of Finance 40, 793-808.

DeLong, J. B., Andrei Shleifer, Lawrence Summers and Robert Waldmann (1990), Noise trader risk in financial markets, Journal of Political Economy 98, 703-38.

Fama, Eugene and Kenneth French. (2002) "The Equity Premium," Journal of Finance 57, 2, (2002) 637-659.

Gardiner, C.W., (1985) Handbook of Stochastic Methods, Springer-Verlag.

Hausman, Jerry A.and David A. Wise: A Conditional Probit Model for Qualitative Choice: Discrete Decisions Recognizing Interdependence and Heterogeneous Preferences, Econometrica, Vol. 46, No. 2 (Mar., 1978), pp. 403-426.

Jegadeesh, N., and S. Titman, 1993, "Returns to buying winners and selling losers: Implications for stock market efficiency,” Journal of Finance 48, 65-91.

Lintner, John (1969), The Aggregation of Investor's Diverse Judgments and Preferences in Purely Competitive Security Markets, The Journal of Financial and Quantitative Analysis, Vol. 4, No. 4., pp. 347-400. 
Lee, Charles M. C., James Myers and Bhaskaran Swaminathan (1999), "What is the Intrinsic Value of the Dow?" Journal of Finance. 54, pp.1693-1741.

McFadden, Daniel, "Conditional Logit Analysis and Qualita- tive Choice ehavior," in Paul Zarembka (ed.), Frontiers in Econometrics (New York: Academic Press, 1974).

Mossin, J. (1966), Equilibrium in a Capital sset Market, Econometrica, Vol. 34, No. 4, pp. $768-783$.

Haruvy, Ernan, E., Lahav, Y., and C. Noussair (2007), Traders' Expectations in Asset Markets: Experimental Evidence, forthcoming in the American Economic Review.

Smith, V. L., Suchanek, G. L., and Williams, A. W., (1988), Bubbles, Crashes and Endogenous Expectations in Experimental Spot Asset Markets, Econometrica, 56(5), pp. 1119-1151.

Battalio, Robert and P. Shultz (2006), Options and the Bubble, Journal of Finance 59-5 2017-2102.

Ofek, Eli, and Matthew Richardson, 2003, DotCom Mania: The rise and fall of Internet stock prices, Journal of Finance 58, 1113-1137.

Robin Greenwood, R., and Stefan Nagel, (2008), Inexperienced Investors and Bubbles, Working Paper 14111, and Journal of Financial Economics (forthcoming).

Weidlich, W., and Haag, G. , 1983, Concepts and Models of a Quantitative Sociology, Springer-Verlag Berlin Heidelberg New York. 


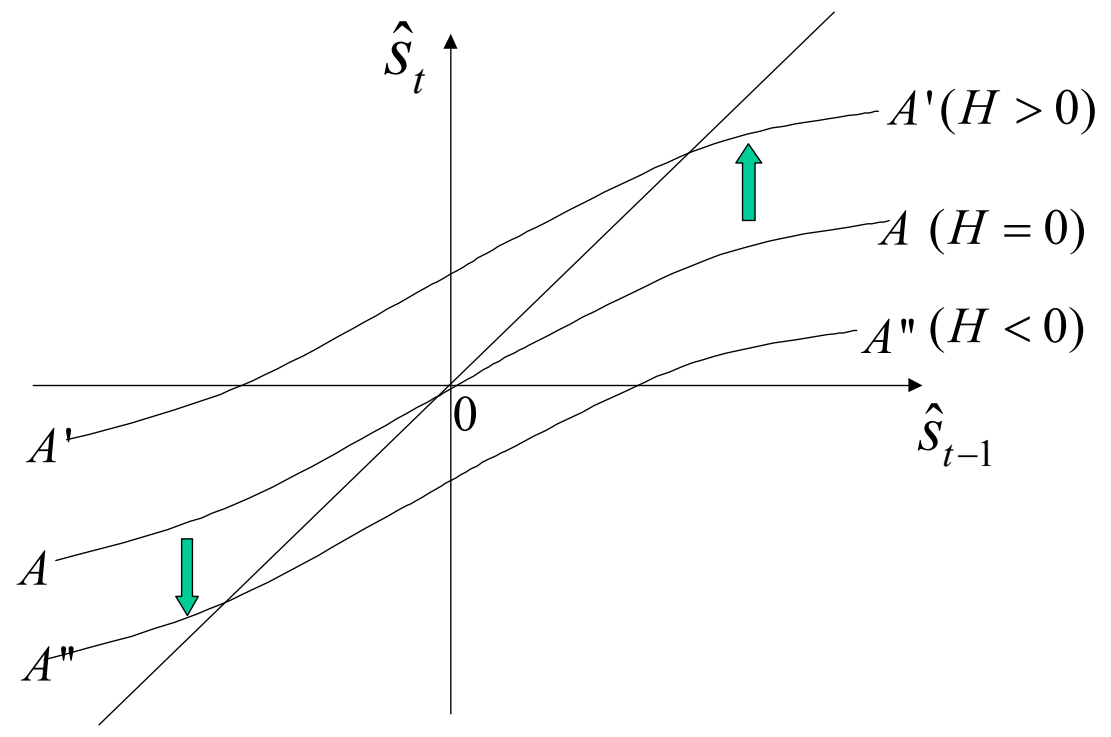

Figure 1: The solutions of the equation (17) for $\lambda<1$ and the three values of $H$. The straight line is $\mathbf{4 5}$ degree line.

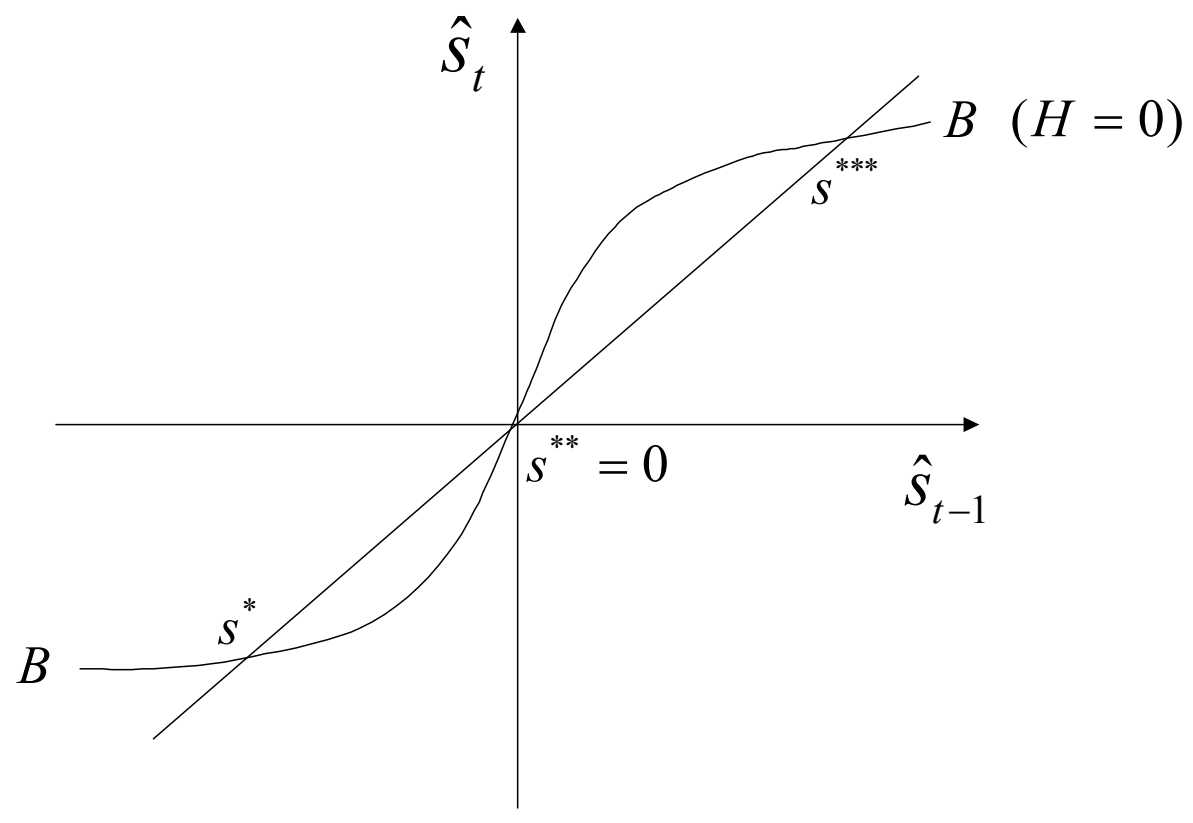

Figure 2: The solutions of the equation (17) for $\lambda>1$ and $H=0$. The straight line is 45 degree line. 


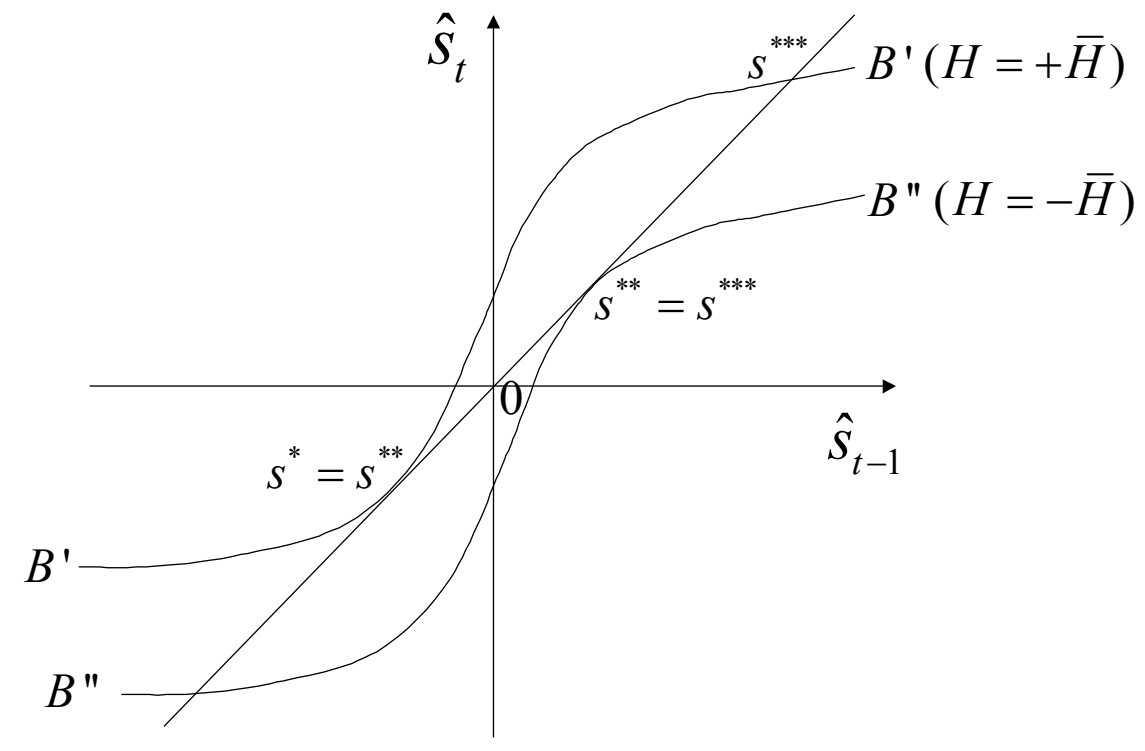

Figure 3: The solutions of the equation (17) for $\lambda>1$ and $H= \pm \bar{H}$. The straight line is 45 degree line.

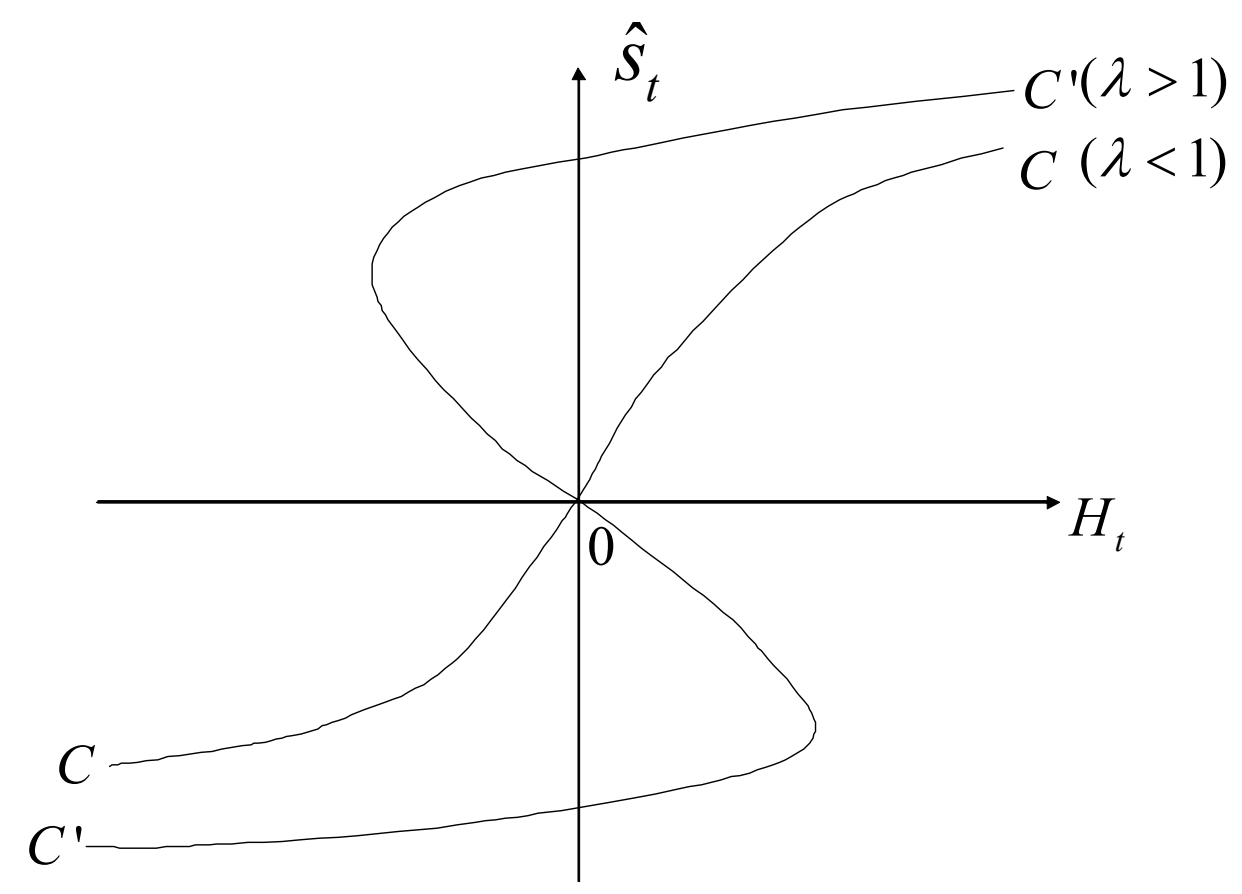

Figure 4: The solutions of the equation (17) for $\lambda>1$ and $\lambda<1$. The figure indicates the relationship of $H$ to the equiribria. 


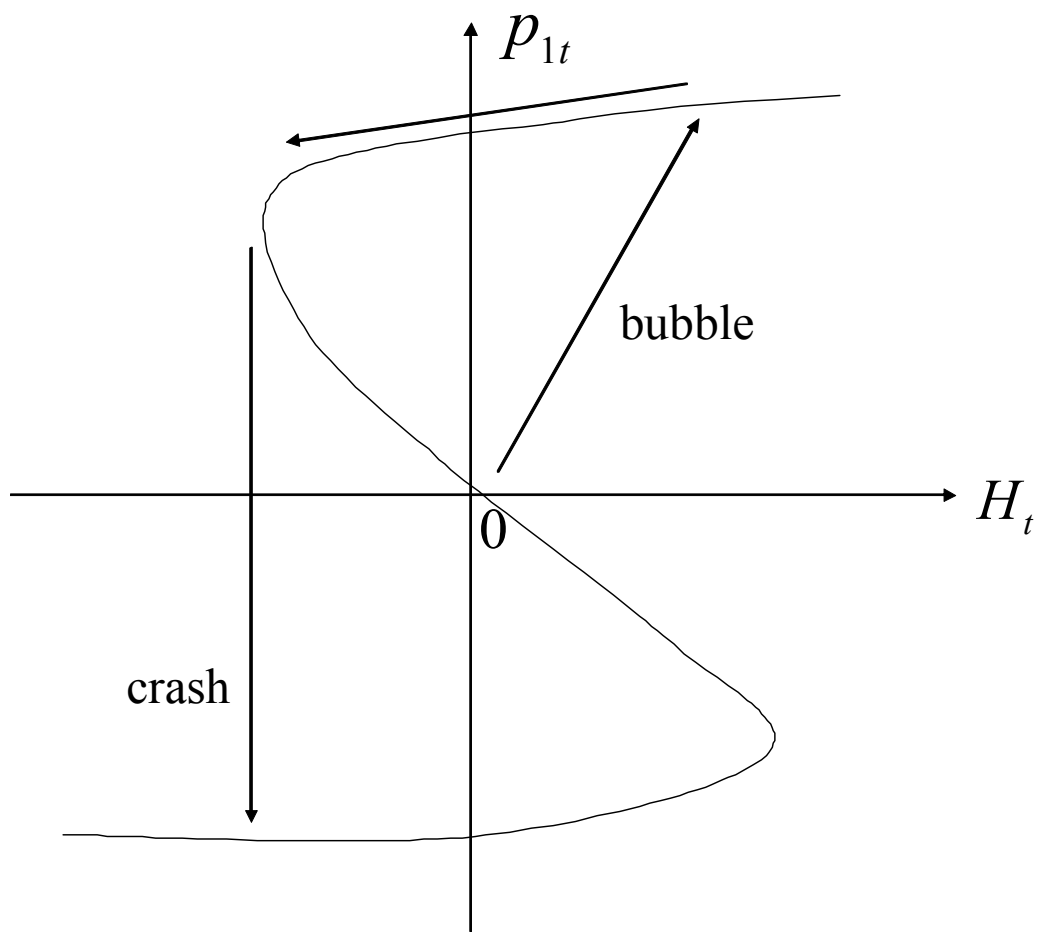

Figure 5: The dynamics of (20) for $\lambda>1$ and $E\left(\Delta p_{j t+1}\right)=0$. Bubble and Crash. 